\title{
GATA-binding protein 1 is a novel transcription regulator of peroxiredoxin 5 in human breast cancer cells
}

\author{
MIN JI SEO ${ }^{1}$, XIYUAN LIU ${ }^{1}$, MINSUN CHANG ${ }^{2}$ and JONG HOON PARK ${ }^{1}$ \\ Departments of ${ }^{1}$ Biological Science and ${ }^{2}$ Medical and Pharmaceutical Science, Sookmyung Women's \\ University, 52 Hyochangwon Road, Yongsan-gu, Seoul 140-742, Republic of Korea
}

Received August 8, 2011; Accepted September 28, 2011

DOI: 10.3892/ijo.2011.1236

\begin{abstract}
Peroxiredoxin 5 (Prx5) is a member of a family consisting of six antioxidant enzymes. Prx 5 is ubiquitously expressed in various tissues including mitochondria and peroxisomes, implying that $\operatorname{Prx} 5$ functions as a regulator of the cellular oxidation state. Prx 5 plays a critical role in protecting cells from oxidative stress by inhibiting the accumulation of reactive oxygen species and cell death. Overexpression of Prx 5 in mammary tissue is associated with poor prognosis of breast cancer. The present study was conducted to elucidate the regulatory mechanisms of Prx 5 gene expression and the physiological relevance of Prx 5 in breast cancer cell survival. Analysis of the promoter region of the Prx 5 gene and reporter gene assays revealed the promoter region critical for Prx 5 gene regulation to which the novel negative transcription regulator, GATA1 binds in human breast cancer cell lines. GATA1 was further confirmed as a potential transcription factor for Prx 5 gene expression in another set of transfection assays using a reporter gene construct that contained a mutated GATA1 binding site. Direct binding of GATA1 to the Prx 5 promoter was demonstrated using chromatin immunoprecipitation and electrophoretic mobility shift assays. Finally, functions of Prx 5 associated with apoptosis were examined in two human breast cancer cell lines displaying high levels of either Prx 5 or GATA1 expression. Knockdown of GATA1 led to increased expression of Prx5 and inhibition of apoptosis. Our data suggest that Prx5 may protect cells from oxidative stress-mediated apoptosis in a GATA1-regulated manner.
\end{abstract}

Correspondence to: Dr Jong Hoon Park, Department of Biological Science, Sookmyung Women's University, Seoul 140-742, Republic of Korea

E-mail: parkjh@sookmyung.ac.kr

Dr Minsun Chang, Department of Medical and Pharmaceutical Science, Sookmyung Women's University, Seoul 140-742, Republic of Korea

E-mail: minsunchang@sookmyung.ac.kr

Key words: peroxiredoxin 5, GATA1, breast cancer, apoptosis, transcription factor

\section{Introduction}

Peroxiredoxins (Prxs) are a family of antioxidant enzymes that regulate the levels of reactive oxygen species (ROS) in cells (1). Since ROS are involved in necrotic or apoptotic cell death as well as the control of signal transduction, critical roles of Prxs include protecting cells against oxidative stress as well as serving as redox regulators of signal transduction $(2,3)$. Knock-out of Prxs 1, 2, 3, or 6 in various animal or cell models results in abnormal physiological conditions such as anemia, malignancies, disruption of hematologic homeostasis, increased susceptibility to pro-inflammatory responses, shortened life span, and accelerated senescence (4-8). It has been noted that such effects are rescued upon overexpression of Prxs or catalase, implying a role of antioxidant Prxs and hydrogen peroxide $\left(\mathrm{H}_{2} \mathrm{O}_{2}\right)(4,9,10)$.

There are six mammalian Prxs which all contain the conserved cysteine residue(s) responsible for peroxide scavenging. Prx1 through Prx5 have two catalytically-active cysteine residues whereas Prx6 has only one cysteine residue. The human Prx5 gene was cloned in 1997 and the full cDNA of this gene was isolated and sequenced in $1999(11,12)$. Crystal structure studies and site-directed mutagenesis of human Prx5 suggested the functional importance of two cysteines (Cys47 and Cys151) as well as the role of Prx5 as an antioxidant $(2,13)$. Human Prx5 gene organization as well as its promoter have been studied (14) and the expression of Prx 5 in concert with Prx1 is regulated by interaction between the Ets transcription factor and high mobility protein group B1 in prostate cancer cells (15). However, the promoter-driven regulatory mechanisms of Prx 5 gene and endogenous factors that modulate Prx 5 gene expression remain unclear.

Prx5 is found in various cell compartments including the cytosol as well as the major intracellular sources of ROS, namely mitochondria and peroxisomes, suggesting that it may have an important role as a signaling molecule and antioxidant in organelles (14). The biological roles of Prx 5 have been defined by its ability to prevent p53-induced apoptosis (3), inhibit various types of DNA damage $(16,17)$, and suppress TNF- $\alpha$ mediated accumulation of $\mathrm{H}_{2} \mathrm{O}_{2}$ (2). Overexpression of Prx5 in lung cancer cells has been associated with the protection of cells from apoptosis, implying that Prx5 prevents apoptosis or oxidative damage (18). In addition, the physiological roles of Prx5 were examined in Drosophila where Prx5 is thought to 
be involved in the modulation of immune responses and protection against oxidative stress that may lead to longevity $(7,19)$. Interestingly, expression of Prx5 was shown to be dependent on tissue type and grade of breast and its expression was induced in response to ROS, indicating that Prx5 is involved in mammary tumorigenesis (20). The mechanistic roles and pathways of Prx5 in gene regulation, apoptosis, and carcinogenesis should be studied further to provide insights into the pathophysiological roles of Prx 5 in breast cancer cells and tissues.

Our present study investigated the role of Prx5 in cell survival and apoptosis in human breast cancer cell lines and a novel regulatory mechanism of Prx 5 gene expression. Our results showed that expression Prx 5 gene was breast cancer cell line-specific. MCF7 cells resistant to the chemotherapeutic agent adriamycin MCF7-Adr showed high expression levels of Prx5 whereas MDA-MB-231 cells had low Prx5 expression levels. Both cell types are associated with multi-drug resistance and serious malignancies compared to other breast cancer cell lines (21). Analysis of the Prx5 promoter region revealed that the 500 bp core region was responsible for gene regulation and identified as the transcription regulator of the Prx 5 gene in these two cell lines. We demonstrated that Prx 5 may help protect cells against oxidative stress-mediated apoptosis in a DNA binding factor (GATA1)-dependent manner. In addition, we suggest that GATA1 acts as a transcription repressor of the Prx 5 gene and that GATA1 is associated with Prx 5 during apoptosis in a human breast cancer cell model.

\section{Materials and methods}

Cell culture and reagents. MCF7s cell line is a steroid-sensitive sub-line developed from MCF7 human breast cancer cells and was provided by Dr Robert Clarke (Lombardi Cancer Center, Georgetown University, Washington D.C. USA) (22). It is grown in cultured in RPMI-1640 supplemented with $10 \%$ fetal bovine serum (FBS) and 1\% antibiotic-antimycotic solution. MCF7-Adr cell line, a MCF7-derived line resistant to adriamycin, has been previously characterized (23). Both MCF7-Adr and MDA-MB231 (American Tissue Culture Collection, GA, USA) cells were grown in DMEM supplemented with $10 \%$ FBS and $1 \%$ antibiotic-antimycotic solution. Cells were maintained in a humidified $5 \% \mathrm{CO}_{2}$ atmosphere at $37^{\circ} \mathrm{C}$. All cell culture reagents were purchased from WelGENE (Daejeon, South Korea). Restriction endonucleases and T4 DNA ligase were purchased from Fermentas MBI (Amherst, NY, USA). Pfu polymerase was obtained from Takara Bio Inc. (Shiga, Japan). Oligonucleotides were synthesized by GenoTech (Daejeon, South Korea). Other molecular biology reagents were purchased from Sigma-Aldrich (St. Louis, MO, USA) unless stated otherwise.

Plasmid construction. The 3000 bp Prx 5 promoter region was amplified by polymerase chain reaction (PCR) using the forward primer 5'-GAAGATCTGACAGCCGACGCA ACTACAAATG-3' and reverse primer 5'-CCCAAGCTT TCGCTGTCGCTGTCTGACCAGATGTC-3'. The PCR product was isolated by gel electrophoresis and digested with $B g l \mathrm{II}$ and HindIII (sites underlined in the primers) to generate the sticky ends for ligation with the expression vector. The final product was subcloned into an upstream portion of the luciferase reporter gene in the pGL3-basic vector (Promega,
Madison, WI, USA). The resulting construct was designated as pGL3-p3000. A series of reporter gene plasmids containing various portion of the $3000 \mathrm{bp} \operatorname{Prx} 5$ promoter region were generated using pGL3-p3000 as a template. The forward PCR primer sequences used for each promoter-reporter construct are listed in a Table I. The number shown in the designation of each plasmid represents the approximate distance from the translation start site in the Prx5 gene. In addition, a pGL3-F500 construct which contains 500 bp upstream of the translation start site in the Prx5 gene was generated using pGL3-p3000 as template plasmid DNA with the following primers; 5'-GAAGATCTACATGCGAGCTCAGCAGATTGTGGG-3' (forward) and 5'-GGGAAGCTTCGCCTCCACTGCGCAGGC GCGAGC-3' (reverse).

Site-directed mutagenesis. The promoter regions of three target genes, Ets-like gene 1 (ELK1), NF-E2-related factor 2 (Nrf2), and GATA-binding protein 1 (GATAl), were subjected to site-directed mutagenesis to study the function of each gene as potential Prx5 gene regulators. Site-directed mutagenesis was performed using Quikchange site-directed mutagenesis kit (Stratagene, La Jolla, CA, USA) according to the manufacturer's instructions. Three constructs containing mutated sequences in the transcription binding sites were generated to prevent each transcription factor from binding to the specific region of the Prx5 promoter region using the primers listed in Table II. The mutated sites are underlined as follows: mtELK1 $($ AACC $\underline{G A A G T} \rightarrow$ AACCTTAAGT), mtNrf2 (CC $\underline{\text { GGAAG }}$ CGTCT $\rightarrow$ CCTTCCGCGTCT), and mtGATA1 (GGGATAG $\mathrm{AC} \rightarrow \mathrm{GGG} \underline{\underline{A T T C A C}) .}$

Transfection and luciferase reporter assay. Luciferase transcription was quantitated by transient transfection of various pGL3 constructs containing the respective DNA sequences corresponding to the promoter regions of Prx5 gene as well as the firefly luciferase reporter gene. Cells were seeded at $4 \times 10^{5}$ cells/well in a 6-well plate and the appropriate pGL3 plasmid (2 $\mu \mathrm{g} /$ well) was transfected into the cells $24 \mathrm{~h}$ after seeding using FuGENE HD transfection reagent (Roche, Basel, Switzerland) according to the manufacturer's instructions. The cells were cotransfected with a phRL-CMV plasmid (Promega) encoding Renilla luciferase to normalize luciferase activity. Luciferase activity $24 \mathrm{~h}$ after transfection was measured using a Dual Luciferase Assay system (Promega) according to the manufacturer's instructions with a Victor $3^{\mathrm{TM}}$ (PerkinElmer, Waltham, MA, USA) in a 96-well plate format. Data are reported as the relative luciferase activity calculated by dividing firefly luciferase activity by Renilla luciferase activity.

Reverse transcription polymerase chain reaction (RT-PCR). Total cellular RNA was extracted and reverse transcribed as described above. The primers used for semi-quantification of the human-specific genes were as follows: GATA1, 5'-CTTTCA GGTGTACCCATTGCT-3' (forward) and 5'-TAGGTAGTG GCCTGTCCTGTC-3' (reverse); Prx5, 5'-ACGGTGCAGTGA AGGAGAGT-3' (forward) and 5'-ACATTCAGGGCCTTCACT ATG-3' (reverse); and GAPDH, 5'-CCATCCAATCGGTAG TAGCG-3' (forward) and 5'-GTAACCCGTTGAACCCCATT-3' (reverse). PCR was performed with a GeneAmp PCR system 
Table I. Sequence of the primers for generating the reporter plasmids containing various portions of $3000 \mathrm{bp}$ upstream of the Prx5 gene.

Designation of the promoter-reporter plasmid

pGL3-p2500

pGL3-p2000

pGL3-p1500

pGL3-p1000

pGL3-p500
Sequence $\left(5^{\prime} \rightarrow 3^{\prime}\right)$

GAAGATCTGCTTACCCACAATCTGCTGTC
GAAGATCTCGCACTGAGGCTGCCAGTGCTCA
GAAGATCTCTGAGCAGGCTCGTTGGGAGCTGAT
GAAGATCTATGCTAGGCAGGTCCTGCCAGAAC
GAAGATCTACCTTGTACCCTCCCCACCTT

Table II. Sequence of the primers used to generate the mutated binding sites in the candidate transcription factors involved in Prx5 regulation.

\begin{tabular}{lll}
\hline Transcription factor & Orientation & Sequence $\left(5^{\prime} \rightarrow 3^{\prime}\right)$ \\
\hline ELK1 & Forward & GGATAGCCAGGAGAACCTTAAGTGGCGAACTTGCTG \\
& Reverse & CAGCAAGTTCGCCACTTAAGGTTCTCCTGGCTATCC \\
Nrf2 & Forward & GTAACGCGCCGCTACCTTCCGCGTCTCAGCAGGAGG \\
& Reverse & CCTCCTGCTGAGACGCGGAAGGTAGCGGCGCGTGAC \\
GATA1 & Forward & CCGTCTTTATGGCGGGATTCACATTTAATCCGGGTACC \\
& Reverse & GGTACCCGGATTAAATGTGAATCCCGCCATAAAGACGG \\
\hline
\end{tabular}

2700 thermal cycler (Applied Biosystems). Amplification conditions were 30 cycles of $94^{\circ} \mathrm{C}$ for $1 \mathrm{~min}, 58^{\circ} \mathrm{C}$ for $1 \mathrm{~min}$, and $72^{\circ} \mathrm{C}$ for $1 \mathrm{~min}$. The amplified products were separated and visualized by electrophoresis on a $1 \%$ agarose gel.

Total RNA isolation and quantitative real-time RT-PCR ( $q R T$ $P C R$ ) analysis. Total cellular RNA was extracted from MCF7-Adr or MDA-MB-231 cells with Nucleospin ${ }^{\circledR}$ RNA/Protein extraction kit (Macherey-Nagel, Düren, Germany) according to the manufacturer's instructions. A total of $2 \mu \mathrm{g}$ of RNA was subject to reverse transcribed using an ImProm-II ${ }^{\mathrm{TM}}$ Reverse Transcription System (Promega) to synthesize cDNA. The resulting cDNA was subsequently quantified by real-time PCR amplification with a Rotor-Gene 3000 (Corbett Research, Sydney, Australia). The sequences of the primers for quantitative real-time PCR were as follows: GATA1, 5'-GTAGCGGGAATTGTGGGGAGGTG-3' (forward) and 5'-GGGAAAGGCATGAGGTGGCTAACA-3' (reverse); Prx 5, 5'-CCACAAGGCGGAAGGCAAGGT-3' (forward) and 5'-TGAGACGTCGATTCCCAAAGATG-3' (reverse); and glyceraldehyde 3-phosphate dehydrogenase (GAPDH) 5'-ATCGTGGAAGGACTCATGACCACA-3' (forward) and 5'-AGAGGCAGGGATGATGTTCTGGA-3' (reverse). Quantitative real-time PCR was performed in a reaction mixture containing 1X SYBR Green PCR Master Mix (Applied Biosystems, Carlsbad, CA, USA) and $0.1 \mu \mathrm{M}$ of each primer. Cycling conditions were one cycle at $50^{\circ} \mathrm{C}$ for $2 \mathrm{~min}$, one cycle at $95^{\circ} \mathrm{C}$ for $10 \mathrm{~min}$, and 40 cycles including a denaturing step at $95^{\circ} \mathrm{C}$ for $15 \mathrm{sec}$ and an annealing-elongation step at $60^{\circ} \mathrm{C}$ for $1 \mathrm{~min}$. Rotor-Gene 6.1 software (Corbett Research) was used to estimate the CT parameter. The $2^{-\Delta \Delta C}{ }_{T}$ method was used to quantify the expression of selected genes; GAPDH was used as housekeeping gene for normalization.
Chromatin immunoprecipitation (ChIP) assay. AChIP assay was carried out using an Imprint Chromatin Immunoprecipitation kit (Sigma-Aldrich) according to the manufacturer's instructions. Briefly, nuclear extracts were isolated from breast cancer cells using a Nuclear Extraction kit from Panomics (Santa Clara, CA, USA) and sonicated to produce 250 to $500 \mathrm{bp}$ chromatin fragments. The sonicated nuclear extracts were incubated with GATA1 antibody (Cell Signaling Technology, Danvers, MA, USA) for 90 min. Mouse immunoglobulin (Ig) $\mathrm{G}$ (Sigma-Aldrich) was used as a control to monitor nonspecific interactions and anti-RNA polymerase II antibody (SigmaAldrich) was used as a positive control. Antibody-GATA1-DNA complexes were pulled down after extensive washing to reduce non-specific binding between proteins and antibodies. The DNA was isolated from protein-DNA complexes by incubating samples at $65^{\circ} \mathrm{C}$ for $16 \mathrm{~h}$. The final DNA was purified using a QIAquick PCR purification kit (Qiagen, Valencia, CA, USA) and resuspended in water for subsequent PCR. The GATA1 binding region in the Prx 5 promoter was amplified using a pair of primers: 5'-TACTCCTACACAGGCGCCTAA-3' (forward) and reverse 5'-TTTGGGCACTGGAGCAGCTA-3' (reverse). The final PCR products were visualized on a $0.8 \%$ agarose gel.

Electrophoretic mobility shift assay (EMSA). Nuclear extracts from MCF7s or MDA-MB-231 cells were isolated using a Nuclear Extraction kit (Panomics). For the EMSA assay, wild-type (wt) GATA1 probes (forward; 5'-AATACCGCCC TATCTGTAAATTAGG-3' and reverse, 5'-CCTAATTTACAG ATAGGGCGGTAT T-3'), mutant (mt) GATA1 probes (forward; 5'-AATACCGCCCTAAGTAAATTAGG-3' and reverse, 5'-CCTAATTTACTTAGGGCGGTATT-3'), and consensus probes (forward; 5'-CACTTGATAACAGAAAGTGATAAC 
A

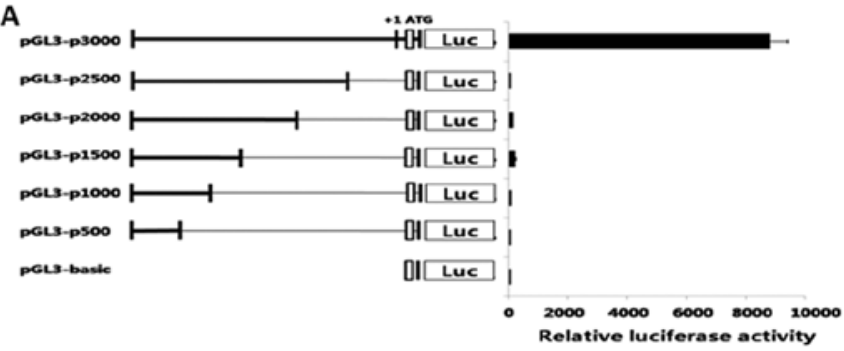

C

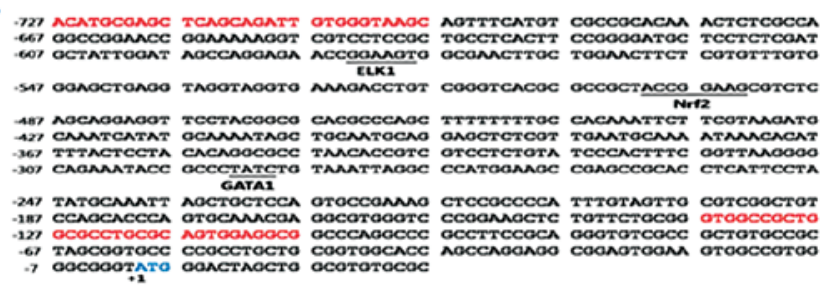

B

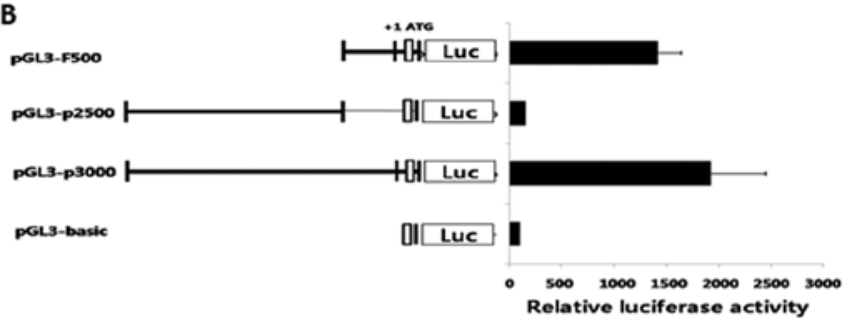

D

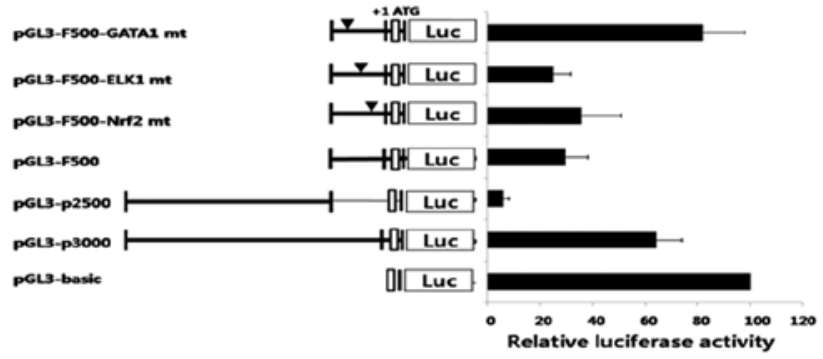

Figure 1. Identification of the Prx5 promoter region in MCF7s cells. (A) MCF7s cells were transfected with a series of Prx 5 promoter reporter constructs. The Renilla phRL-CMV vector was used to normalize the transfection efficiency. The reporter plasmid pGL3-p3000 indicated the size of the Prx5 promoter region inserted to the pGL3-basic vector. Luciferase activity of the pGL3-basic empty vector was used as a control. (B) The 500 bp region between pGL3-p3000 and pGL3-p2500 was subcloned into the pGL3-basic vector to confirm the recovery of luciferase activity. The reporter plasmid pGL3-F500 indicated the region between pGL3-p3000 and pGL3-p2500. (C) The 500 bp region upstream of the Prx5 translation start site subcloned as pGL3-F500 was analyzed using a promoter prediction program. The translation start codon, ATG, is indicated in blue. The sequences denoted in red span the 500-bp nucleotides in the pGL3-F500 reporter plasmid. (D) The three binding sites of the predicted transcription factors were targeted for site-directed mutagenesis. These mutational constructs were transfected into MCF7s cells. The Renilla phRL-CMV vector was co-transfected to normalize the transfection efficiency.

TCT-3' and reverse 5'-AGAGTTATCACTTTCTGTTATCAA GTG-3') were labeled by combining $250 \mathrm{ng}$ of the annealed oligonucleotide; $2 \mu \mathrm{l}$ of $10 \mathrm{x}$ T4 kinase buffer (Takara Bio); $1 \mu \mathrm{l}$ each of $5 \mathrm{mM}$ stock of dATP, dTTP, and dGTP; $8 \mu \mathrm{l}$ of water; 1 $\mu l$ of $\mathrm{T} 4$ polynucleotide kinase (Takara Bio) and $5 \mu \mathrm{Ci}$ of $\left[{ }^{32} \mathrm{P}\right]-$ $\mathrm{dCTP}$ (Amersham Biosciences) at $37^{\circ} \mathrm{C}$ for $1 \mathrm{~h}$. The labeled probe was isolated using Microspin columns (Bio-Rad, Hercules, CA, USA). A typical reaction consisted of $10 \mathrm{ng}$ of nuclear protein extracts, $250 \mu \mathrm{g} / \mathrm{ml}$ poly(deoxyinosinic-deoxycytidylic acid), and radiolabeled double-stranded oligonucleotides in a solution of $30 \mathrm{mM}$ HEPES, $100 \mathrm{mM} \mathrm{KCl}, 1.5 \mathrm{mM} \mathrm{MgCl}_{2}, 10 \%$ glycerol, $1 \mathrm{mM}$ dithiothreitol, $0.3 \mathrm{mM}$ EDTA, and protease inhibitor (Sigma-Aldrich). Binding between oligonucleotides and GATA1 was induced by incubating samples at $37^{\circ} \mathrm{C}$ for $20 \mathrm{~min}$. Final binding complexes were loaded onto $6 \%$ polyacrylamide gels and the gel was dried, and separated at $150 \mathrm{~V}$ for $2.5 \mathrm{~h}$. Competition experiments were performed by adding a 100 -fold excess of unlabeled oligonucleotides. The gel was exposed to Fuji film (Tokyo, Japan) at $25^{\circ} \mathrm{C}$ for $24 \mathrm{~h}$ and visualized by a FLA-7000 phosphoimager (Fuji Film Life Science, Tokyo, Japan).

Western blot analysis. Total cellular protein was extracted with Nucleospin ${ }^{\circledR}$ RNA/Protein extraction kit according to the manufacturer's instructions. Protein concentrations were measured with a bicinchoninic acid assay kit (Promega). Equal amounts of protein were separated by $12 \%$ sodium dodecyl sulfate-polyacrylamide gel electrophoresis (SDS-PAGE) and transferred onto a nitrocellulose membrane (Amersham Biosciences, Buckinghamshire, UK). The membrane was probed with the primary antibody against each of the following protein; GATA1, Prx5, Bax, Bcl-2 (Santa Cruz Biotechnology, Santa Cruz, CA, USA), and $\beta$-actin (sc-15, Sigma-Aldrich). Secondary goat anti-rabbit IgG and goat anti-mouse IgG were purchased from Sigma-Aldrich. The blotted proteins were visualized using an enhanced chemiluminescence (ECL)-Plus Western blotting detection system (Amersham Biosciences) and images were captured with an LAS-3000 (Fujifilm Life Science). Band intensities were quantitated with a Multi Gauge 3.0 software image reader (Fujifilm Life Science).

Small interfering RNA (siRNA) transfection. siRNAs targeting either GATA1 or Prx 5 were obtained from Santa Cruz Biotechnology. siRNA was transfected into MCF7-Adr or MDA-MB-231 cells using Lipofectamine RNAiMAX reagent (Invitrogen, Carlsbad, CA, USA) according to the manufacturer's instructions a day after cells were seeded in $100 \mathrm{~cm}^{2}$ culture dishes. Subsequent experiments were performed $24 \mathrm{~h}$ after transfection.

Fluorescence-activated cell sorting (FACS) analysis. Apoptosis was induced in siRNA-transfected cells by exposure to $10 \mathrm{mM}$ of hydrogen peroxide $\left(\mathrm{H}_{2} \mathrm{O}_{2}\right)$. The cells were harvested $24 \mathrm{~h}$ after $\mathrm{H}_{2} \mathrm{O}_{2}$ treatment and washed in phosphate buffered saline (PBS). The cell pellet was resuspended in annexinV binding buffer (BD Bioscience, Franklin Lakes, NJ, USA). Five microliters of annexin V-fluorescein isothiocyanate (FITC) was added and flow cytometry was conducted using a FACS Calibur apparatus (Beckman Instruments, Fullerton, CA, USA). The results were analyzed with CellQuest software (Becton-Dickinson, Heidelberg, Germany). 
A

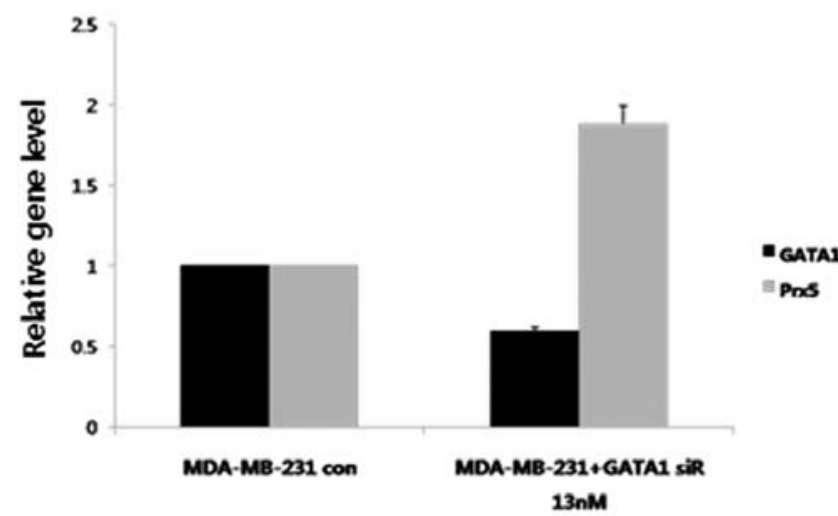

B

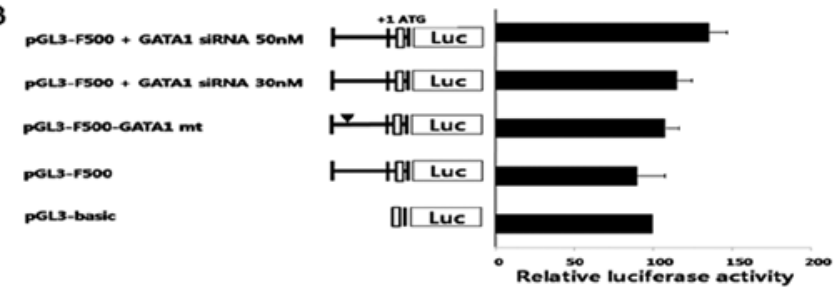

C

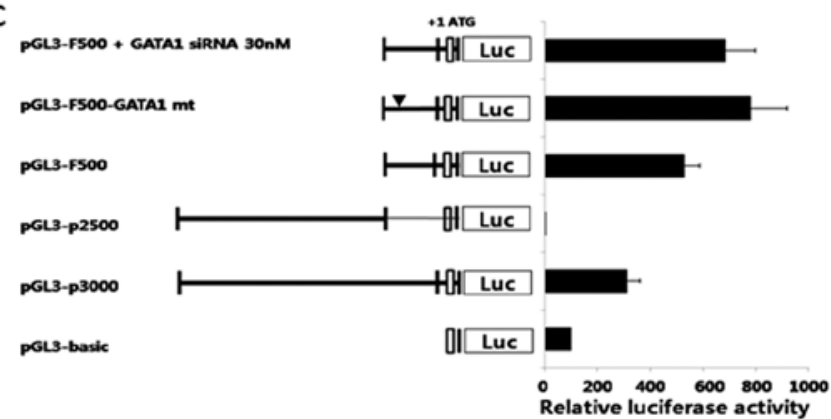

Figure 2. GATA1-mediated modulation of both gene expression and promoter activity of Prx5. (A) GATA1 siRNA (13 nM) was transfected into MDA-MB231 cells, which have a high level of endogenous GATA1 expression, and Prx5 expression in these cells was confirmed using real-time quantitative RT-PCR and compared to those treated with vehicle control ('MDA-MB-231 con' in the figure). GAPDH was used as a control for normalization. (B) MDA-MB-231 cells were seeded in a 6-well plate. Twenty-four hours after transfection with either pGL3-F500 alone, a combination of pGL3-F500 and GATA1 siRNA $(30 \mathrm{nM})$, or pGL3-F500 containing a mutation in the GATA1 binding site (pGL3-F500-GATA1 mt), the cells were harvested and their luciferase activity was determined. (C) pGL3 vectors containing various portions of the Prx5 promoter region were transfected into MCF7s cells and luciferase activities were measured as described in (B).

\section{Results}

Cloning of Prx5 promoter-containing reporter constructs and identification of the core region. The highest luciferase activity was observed in cells transfected with the pGL3-p3000 construct whereas promoter activity of cells transfected with the pGL3-p2500 construct was significantly reduced (Fig. 1A). Luciferase activities of other serial deletion constructs (from pGL3-p2000 to pGL3-p500) were very low and similar to that of the pGL3-2500 construct (Fig. 1A). These data suggested that the region of approximately $500 \mathrm{bp}$ upstream of the translational start site between the regions contained in pGL3-p3000 and pGL3-p2500 was critical for regulating the expression of
A

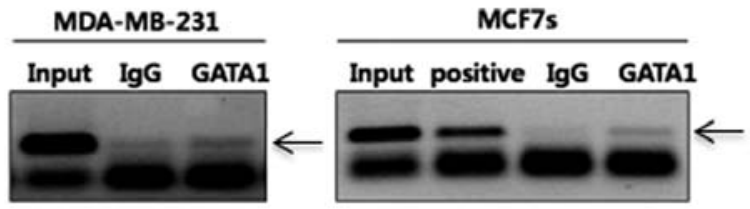

B
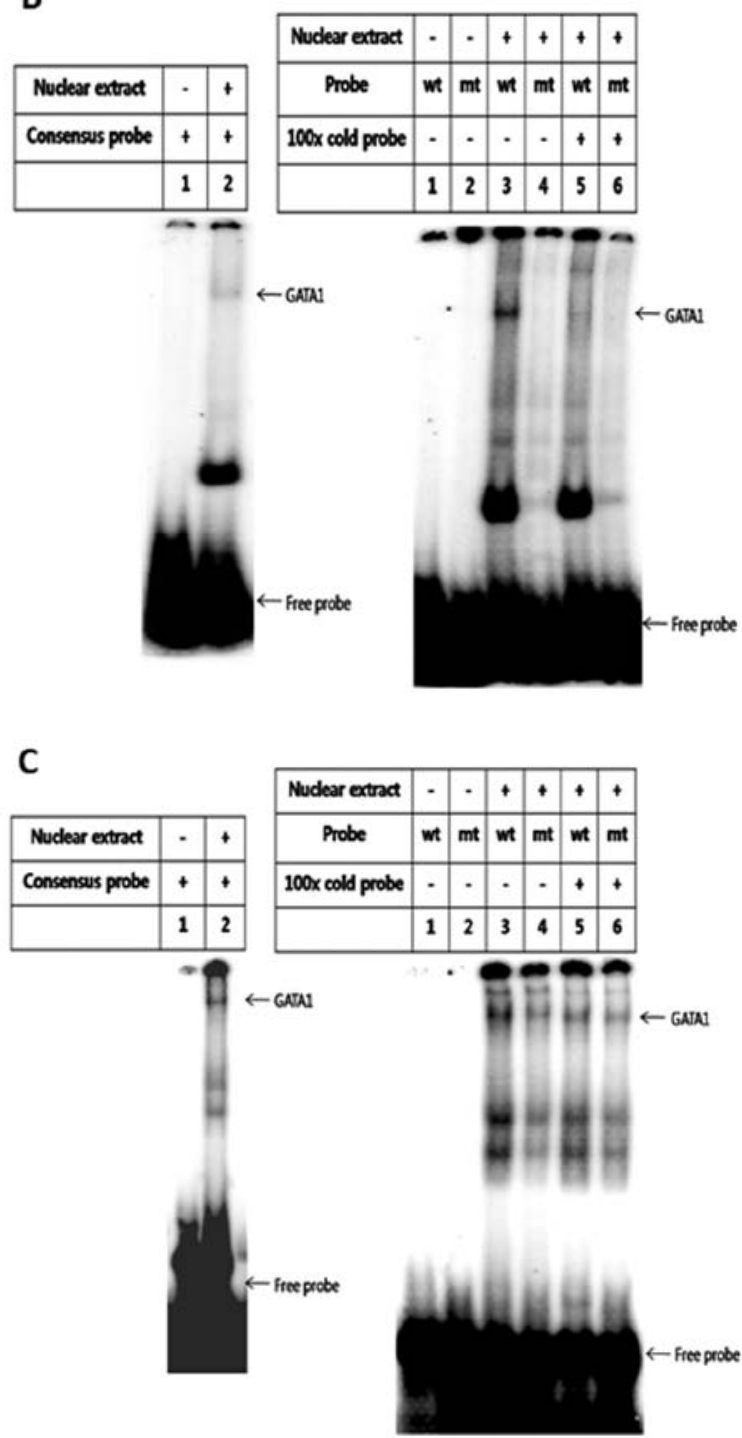

Figure 3. Direct binding of GATA1 to the Prx 5 promoter in cell lysates and cultured breast cancer cells. (A) A ChIP assay was performed in MDA-MB231 cells, which have high endogenous GATA1 expression levels, and in MCF7s cells using the antibodies and primer pairs described in 'Materials and methods'. Binding affinity was measured by PCR. Binding affinity of mouse normal IgG was used as a negative control. An arrow indicates the PCR product corresponding to GATA1 binding site in the Prx5 promoter region. (B) EMSA was carried out in MDA-MB-231 cells using radiolabeled oligonucleotides containing the wild-type or mutated sequences corresponding to the GATA1 binding site of the Prx5 promoter. Unlabeled oligonucleotides containing either wild-type or mutant GATA1 binding sites were used as a specific competitor. (C) EMSA was carried out in MCF7s cells using radiolabeled oligonucleotides containing the GATA1 binding site of the Prx5 promoter.

the Prx 5 gene. To confirm the promoter activity of this core region, a pGL3-F500 construct was generated that contained the 500 bp region of the Prx 5 promoter identified above. Luciferase 
A

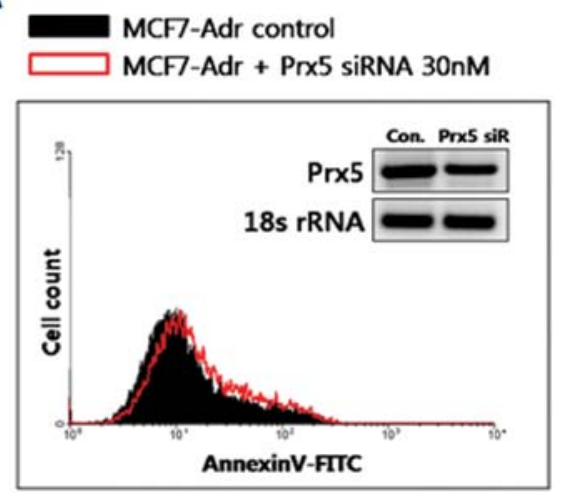

B

C

MDA-MB-231 control

MDA-MB-231 + GATA1 siRNA 40nM
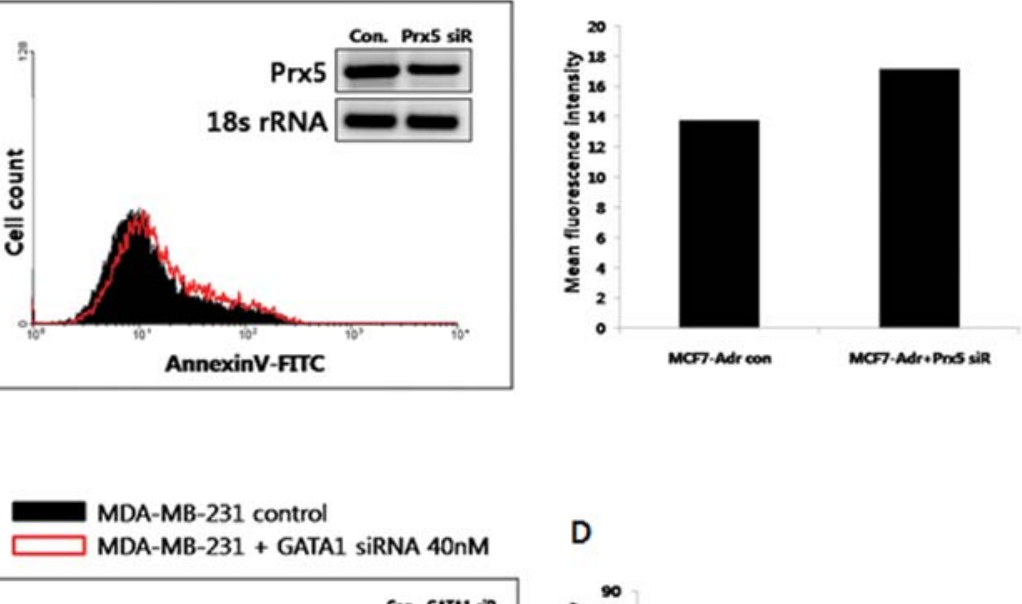

D
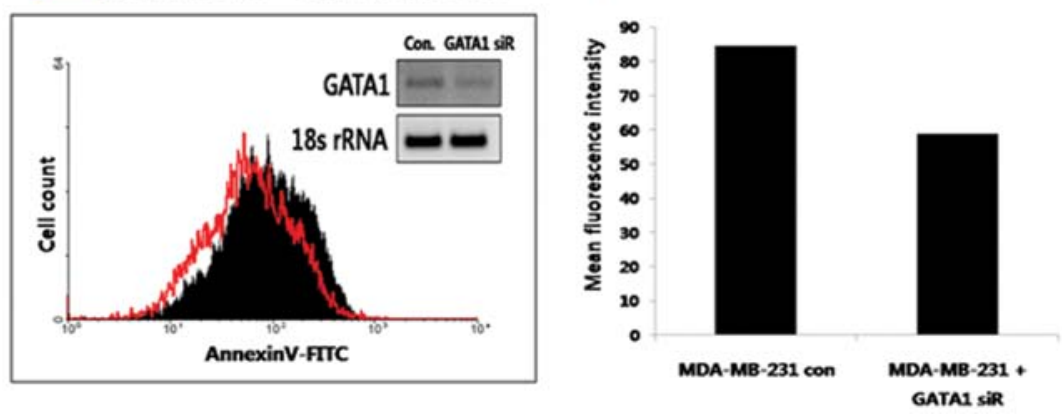

Figure 4. Protection of cells from hydrogen peroxide-induced apoptosis via Prx5 expression (A) Prx5 siRNA was transfected into MCF7-Adr cells, which have high levels of endogenous Prx5. An inset picture shows the relative expression of Prx5 in cells treated with siRNA or no siRNA (control). FACS analysis was performed to measure $\mathrm{H}_{2} \mathrm{O}_{2}$-induced apoptosis in MCF7-Adr cells treated with Prx5 siRNA. Cells not exposed to $\mathrm{H}_{2} \mathrm{O}_{2}$ were used as a control to confirm annexinV staining. (B) Mean fluorescence intensities in MCF7-Adr cells with high or low Prx5 expression produced similar FACS histogram patterns. (C) GATA1 siRNA was transfected to MDA-MB-231 cells and the expression of GATA1 was detected by RT-PCR compared to samples treated with no siRNA (control). FACS analysis was performed as described in (A). (D) Mean fluorescence intensity in MDA-MB-231 cells with high or low GATA1 expression produced similar FACS histogram patterns. All experiments were performed in duplicate.

activity in cells transfected with the pGL3-F500 construct was similar to that shown in cells transfected with pGL3-p2500 and reached $60 \%$ of the maximum promoter activity found in cells expressing the pGL3-p3000 construct (Fig. 1B).

Identification of transcriptional regulators that interact with the promoter core region. To identify transcriptional regulators of Prx 5 that may directly interact with the $500 \mathrm{bp}$ core region, the public prediction program, Transcription Factor Binding Sites Search program (TFSEARCH) version 1.3 (Parallel Application TRC Laboratory, Japan) was utilized. As a result, three candidate genes, ELK1, Nrf2, and GATA1, were selected (Fig. 1C) and the function of each gene was further examined in terms of Prx 5 gene transcription regulation. Additional constructs based on pGL3-F500 were generated that contained mutated nucleotides in regions corresponding to the binding sites that interact with ELK1, Nrf2, or GATA1. Promoter activities were tested in MCF7s cells transfected with pGL3-F500 (wild-type control) or reporter constructs containing the specific mutation, namely pGL3-F500-ELK1mt, pGL3-F500-Nrf2mt, or pGL3F500-GATA1mt. No significant changes in luciferase activities were observed in cells transfected with pGL3-F500-ELK1mt or pGL3-F500-Nrf2mt, whereas a 3-fold increase was observed in cells transfected with pGL3-F500-GATA1mt compared to those expressing the wild-type construct (Fig. 1D). Our results implied that GATA1 plays a key role in modulating expression of the $\operatorname{Prx} 5$ gene as a specific transcription repressor.

Regulatory effects of GATA1 on Prx 5 gene transcription. Once GATA1 was identified as a potential transcription regulator of Prx5, the mode of GATA1 regulatory action was examined. It is notable that the levels of Prx 5 and GATA1 were inversely proportional in two specific breast cancer cell lines we used. For example, MCF7-Adr cells displayed the highest Prx 5 expression and the lowest GATA1 whereas MDA-MB-231 cells showed the opposite Prx 5 and GATA1 expression patterns. Thus, these two cell lines were selected to further confirm the inverse relationship between these two genes and the regulatory effects of GATA1 on Prx 5 expression. The decrease of GATA1 gene expression by siRNA in MDA-MB-231 cells resulted in a twofold increase of Prx5 gene expression as shown in Fig. 2A. Prx5 promoter activities were also recovered in cells transfected with pGL3-F500 in the same cell line, implying that GATA1 may play a role in Prx 5 expression as a negative regulator (Fig. 2B). To confirm this result in another cell line, a similar experiment was performed in MCF7s cells. Prx5 promoter activities were recovered when either the GATA1 binding site was mutated or GATA1 expression was inhibited by siRNA (Fig. 2C). These 

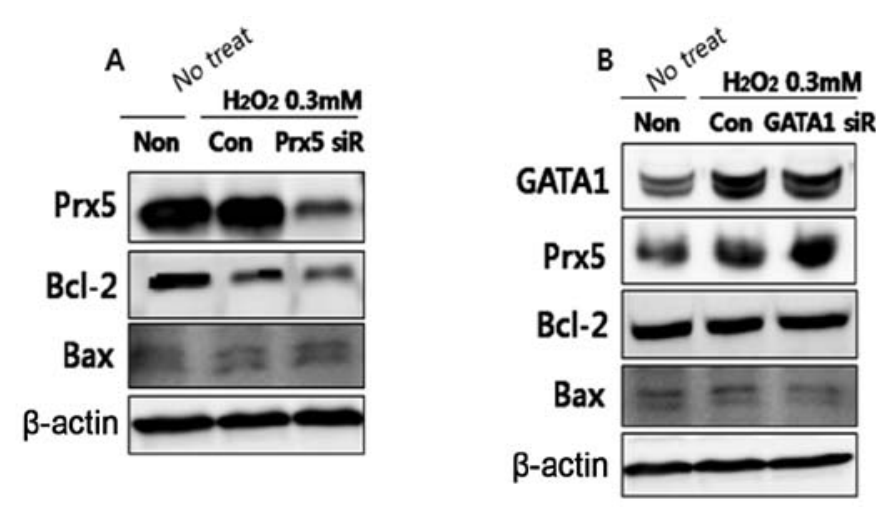

Figure 5. Modulation of apoptotic/anti-apoptotic proteins in response to knockdown of either Prx 5 or GATA1 expression. Samples designated as 'Non' represent those treated with no siRNA. Control samples that were not challenged with $\mathrm{H}_{2} \mathrm{O}_{2}$ are expressed as 'No treat'. (A) Prx5 siRNA was transfected into MCF7-Adr cells. The cells were treated with $\mathrm{H}_{2} \mathrm{O}_{2}$ to induce apoptosis $24 \mathrm{~h}$ after transfection. The expression of Bcl-2, an anti-apoptotic protein, and Bax, a pro-apoptotic protein, were detected by Western blotting. $\beta$-Actin was used as a loading control. (B) GATA1 siRNA was transfected into MDA-MB-231 cells. Protein levels were determined as described in (A).

data confirmed our finding that GATA is a negative transcription regulator of Prx5 gene expression (Fig. 1).

Direct binding of GATA1 to the Prx5 promoter region. Direct and indirect interaction of GATA1 protein with the Prx 5 promoter region was studied using a ChIP assay and an EMSA in MDA-MB-231 and MCF7s cells. A ChIP assay was performed to pull down complexes containing GATA1 protein and the GATA1 binding site of the Prx 5 promoter region using a GATA1-specific antibody and primers specific for the GATA1binding sequence. It was observed that anti-GATA1 antibody had a higher affinity to the chromatin extracts of MDA-MB-231 and MCF7s breast cancer cells than the mouse IgG antibody. This was confirmed by detecting DNA fragments containing the GATA1-binding sequence in the Prx5 promoter region, which were the products of PCR using primers specific for the GATA1-binding region and a template DNA eluted from the chromatin-protein complexes (Fig. 3A). These ChIP results revealed that GATA1 protein could specifically bind to the GATA1-binding sequences in the promoter region of the Prx5 gene.

Direct binding of the GATA1 protein to the Prx5 promoter was further confirmed by an EMSA with lysates from both MDA-MB-231 (Fig. 3B) and MCF7s (Fig. 3C) cells. Nuclear extracts from both cell lines were incubated with oligonucleotide probes containing either the wild-type or mutated GATA1 binding site in the Prx 5 promoter region and were radiolabeled. Only samples containing radiolabeled wild-type probe showed detectable binding affinity in both cell lines (lane 3 in Fig. 3B and C). Binding affinity was reduced when nuclear extracts were incubated with additional excess non-radiolabeled probes (lane 5 in Fig. 3B and C) and mutant probes (lane 2 in Fig. 3B and $\mathrm{C}$ ). These results showed that there is a specific proteinDNA interaction between GATA1 and the GATA1-binding sequence in the Prx 5 promoter.

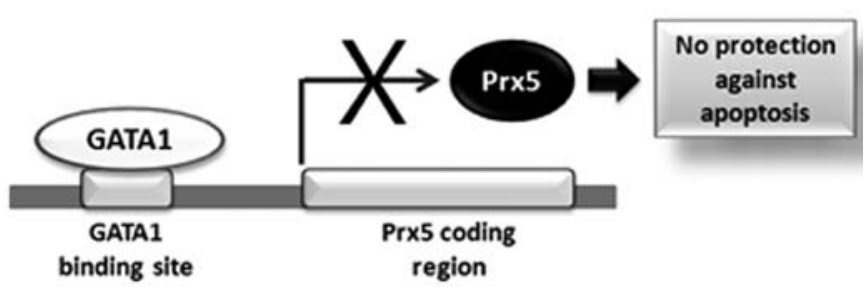

Figure 6. Regulation of Prx5 gene expression via GATA1 and its physiological relevance in breast cancer cells. GATA1 acts as a transcription factor by binding to its binding site in the Prx 5 promoter region and regulating Prx 5 expression in a negative manner. GATA1 binding to the promoter region leads to reduction of transcription of Prx 5 gene and Prx 5 protein expression. Cells under oxidative stress may undergo apoptosis due to loss of Prx5-mediated protection against apoptosis.

Effects of GATA1 on Prx5-mediated apoptosis. Since Prx5 is known to play a protective role in cells under oxidative stress, we examined whether the Prx 5 gene regulated by GATA1 is associated with modulation of apoptosis in both Prx5-sufficient MCF7-Adr cells and GATA1-sufficient MDA-MB-231 cells. MCF7-Adr cells were challenged with $\mathrm{H}_{2} \mathrm{O}_{2}$ for $24 \mathrm{~h}$ to induce apoptosis and expression of Prx 5 was downregulated by siRNA against the Prx5 gene to determine the role of Prx5 in oxidative stress-mediated apoptosis. Knockdown of Prx5 expression increased sensitivity of MCF7-Adr cells to apoptosis as determined by FACS analysis where peak shifts toward the right in the histogram was indicative of increased apoptosis (Fig. 4A and B). FACS analysis was also performed in MDA-MB-231 cells, which have a high level of the endogenous GATA1 expression, to examine the functional relationship between GATA1 and Prx5-associated apoptosis. Reduction in GATA1 expression by siRNA treatment rescued cells from $\mathrm{H}_{2} \mathrm{O}_{2}$-induced death by approximately $35 \%$ compared to non-siRNA treated MDA-MB231 cells as shown in Fig. 4C. These data suggest that Prx5 protects cells from oxidative stress-mediated apoptosis and Prx5-associated apoptosis may be modulated by GATA1 which acts as a negative transcription regulator of the Prx 5 gene.

Mechanistic pathways underlying GATA1 and Prx5-mediated apoptosis. To investigate the mechanistic pathways of Prx5related apoptosis via transcriptional regulation of Prx 5 by GATA1, altered expression of proteins associated with apoptotic pathways were examined. Expression of a pro-apoptotic protein Bax, and the anti-apoptotic factor Bcl-2, were examined by Western blot analysis in cells with reduced levels of either Prx 5 or GATA1 by siRNA and with exposure to $\mathrm{H}_{2} \mathrm{O}_{2}$. When oxidative stress was induced by $\mathrm{H}_{2} \mathrm{O}_{2}$ in MCF7-Adr cells that had been treated with siPrx 5 (siRNA targeting the Prx 5 gene), Bax expression was diminished whereas Bcl-2 levels were elevated compared to cells treated with vehicle control (Fig. 5A). These data along with the results shown in Fig. 4A indicated that Prx 5 inhibits apoptosis by modulating mitochondrial apoptotic proteins such as Bax or Bcl-2. Downregulation of GATA1 by siGATA1 in MDA-MB-231 cells showed the opposite results compared to those observed in MCF7-Adr treated with siPrx5. This led to upregulation of Prx 5 as expected since inhibition of GATA1 would increase the expression of Prx5. In addition, increased levels of Bcl-2 in conjunction with Bax downregulation (Fig. 5B) may be caused by resistance to oxidative stress in 
MDA-MB-231 cells as shown in Fig. 4C. The results shown in Fig. 5A and B implied that Prx5 may affect the mitochondriadependent apoptotic pathway.

\section{Discussion}

Living organisms are exposed to ROS, such as superoxide anions, hydrogen peroxides, organic hydroperoxides, or nitric oxides, which are produced during normal metabolic processes, particularly in the mitochondria $(24,25)$. A low level of ROS is beneficial for normal cellular process including signal transduction, apoptosis, cell differentiation, and regulation of transcription factors $(26,27)$. However, excess ROS can chemically modify cellular macromolecules including DNA, proteins, carbohydrates, or lipids, thereby disrupting the normal physiological functions of these biomolecules (28). Normal or elevated levels of ROS may evoke apoptosis by induction or activation of various apoptotic molecular markers such as cytochrome c, caspases, p53, and c-jun N-terminal kinase $(29,30)$.

ROS are believed to contribute to the development of many types of diseases such as neurodegenerative diseases and cancers $(31,32)$. ROS have been implicated in carcinogenesis by promoting cancer cell invasiveness, genomic instability, cell proliferation/differentiation, and alterations in sensitivity to anticancer drugs (33). Thus, ROS homeostasis is crucial for proper cellular function and survival, and accordingly, mammalian cells have diverse systems to maintain appropriate ROS levels.

Prxs are a family of thiol peroxidase ubiquitously found in all living organisms that reduce hydrogen peroxide and organic hydroperoxides via orchestrated mechanisms among other antioxidants. Among the six Prx isozymes, Prx1 is the most abundant and its biological functions and gene regulation have been extensively examined. For example, a previous study of the regulatory region of the Prx 1 promoter identified Nrf2 as its transcriptional regulator in human lung cancer cells (34). Another study using Prx1 knock-out mouse embryo fibroblasts (MEFs) reported a ROS shift with only Prx5 expression being elevated (35). This study also demonstrated that c-Myc may be a transcriptional factor of Prx 5 and the presence of Prx 1 may repress Prx5 expression, implying that these two factors are functionally related and regulate their own expression. Coordinated gene regulation of Prx1 and 5 has also indicated that expression of Prx 5 as well as Prx 1 could be regulated by interaction between the Ets transcription factor and high mobility protein group B1 in prostate cancer cells (15).

It has been previously shown that Prx 5 can protect cells from oxidative stress by modulating apoptosis in various types of cells such as mouse muscle (36) and human tendon cells (37). Downregulation of Prx5 was shown to affect mitochondria membrane potential and induce apoptosis in small cell lung cancer cells (18). Prx5 was also shown to prevent the p53-dependent formation of ROS and inhibit p53-induced apoptosis in HeLa cells (3). The DNA-specific function of Prx 5 was characterized by the observation that Prx 5 protects genomic integrity from oxidative stress in mammalian cells $(17,18,38)$. These findings suggest that Prx5 is closely involved in modulating intracellular redox cycling and plays a critical role in apoptosis and protection of cells from oxidative stimulus.

It has been reported that expression of Prx 5 in concert with $\operatorname{Prx} 1,3$, and 4 is higher in breast cancer tissues and is signifi- cantly greater if tumors are larger or have lymph node metastases (20). This implies that Prx5 expression is associated with mammary carcinogenesis. However, the specific mechanisms of Prx5 function and expression regulation that are still unclear in developing breast cancer. Protection provided by Prx 5 against oxidative stress and apoptosis in addition to differential Prx5 expression found in various breast cancerous tissues prompted us to examine the transcriptional regulation and function of Prx5 in breast cancer cells.

In the present study, it was found that GATA1 is a novel transcription factor negatively regulating Prx 5 gene expression in breast cancer cell lines. Initial attempts to identify the core region of the Prx 5 promoter were made by analyzing the promoter region starting $3 \mathrm{~kb}$ upstream of the translational start site. Our results showed that a region of approximately $500 \mathrm{bp}$ upstream of the Prx5 gene was sufficient to control gene expression. Interestingly, the binding sites of numerous transcriptional factors including PPAR $\gamma$, Elk1, Oct1, Nrf2, Forkhead box, or GATA1 were found within this $500 \mathrm{bp}$ region using the promoter prediction program, TFSEARCH. Elk1, Nrf2, and GATA1 among the factors selected for further investigation for the following reasons (Fig. 1C). First, Nrf2 is a known transcriptional factor of Prx1 (34). Second, ELK1 is associated with hypoxic conditions and tumorigenesis (39). Finally, GATA1 is a part of an established family of transcriptional factors specifically involved in erythropoiesis (40) and interacts with the estrogen receptor (ER) $(41,42)$.

In order to clarify which transcription factor(s) help in regulating Prx 5 gene expression, a typical reporter gene assay was performed and promoter activities were measured in breast cancer cells that displayed a level of luciferase activity proportional to promoter activities. In addition, promoter activity assays were performed using reporter gene constructs containing mutated nucleotides located in the middle of the binding sites for each potential transcription factor. Promoter activity increased when the lucife-rase reporter construct contained the point mutation site within the GATA binding site was expressed in breast cancer cells (Fig. 1C), thereby indicating that GATA1 is a Prx 5 transcription factor. Additionally, Prx 5 promoter activity was elevated in breast cancer cells in which GATA1 levels were reduced by siRNA to a level similar to that of cells transfected with the reporter gene containing the GATA1 site-directed mutation nucleotide (Fig. 1B and C). Prx5 expression inversely correlated with that of GATA1 (Fig. 5B). The direct binding of GATA1 to the Prx 5 promoter region was confirmed by performing a ChIP assay and EMSA (Fig. 4A-C). All of these data consistently indicated that GATA1 may interact with a specific sequence in the Prx 5 promoter region, and this binding could reduce Prx5 gene expression (Fig. 6).

Prx5 has been shown to be involved in ROS-mediated apoptosis in HeLa and lung carcinoma cells $(3,18)$. We therefore investigated the physiological roles of Prx5 in ROS scavenging and apoptosis in the MCF7-Adr cell line that is ER-positive and has high endogenous expression of Prx5. Our results showed that knockdown of Prx5 expression exacerbated $\mathrm{H}_{2} \mathrm{O}_{2}$-induced apoptosis (Fig. 4A and B). Reduced expression of GATA1 in ER-negative MDA-MB-231 cells led to suppression of negative GATA1 regulatory activity on Prx5 gene and caused decreased apoptosis (Fig. 4C and D). These observations implied that GATA1 indirectly controls oxidative stress-induced apoptosis, 
probably through Prx5 gene regulation (Fig. 6). Additional data demonstrated that modulation of apoptosis by Prx 5 is associated with changes in the expression of proteins that are involved in mitochondria-dependent apoptotic pathways (Fig. 5A and B). These findings are consistent with the localization of Prx 5 in mitochondria. Thus, our data indicate that apoptosis may also be affected by Prx 5 not only in lung or cervical cancer cells, but also in breast cancer cells.

Prx6 is highly expressed in metastatic breast cancer cells compared to breast cancer cells that are less prone to metastasize. Upregulation of Prx6 can increase the proliferation and invasion of breast cancer cells (43). Higher expression of Prx5 in metastatic breast cancer tissues as well as the roles of Prx6 in carcinogenesis prompted us to examine the effects of Prx 5 on breast cancer cell proliferation and invasion. No significant influence of Prx 5 on invasion and metastasis in breast cancer cell lines was observed (data not shown).

GATA1 is the founding member of the GATA factor family of DNA-binding proteins and plays a critical role in erythrocyte development (44). The DNA-binding domains of GATA1 consist of two zinc-finger domains. The carboxyl-terminal zinc finger (GATA1 CF) has a high binding affinity for the A/TGATAA/G consensus sequence. The amino-terminal zinc-finger domain (GATA1 NF) is associated with independent binding to a GAT-containing sequence at a lower affinity $(45,46)$. In addition, both zinc fingers participate in protein-protein interaction with multiple partners such as friend-of-GATA1 (FOG-1) and ER $(41,42,47)$. It has been demonstrated that the ER negatively regulates the transcriptional activity of GATA1 in an ER ligand-dependent manner via physical interaction. ER bound to agonists such as $E_{2}$ are able to directly associate with GATA1 thus inhibiting GATA1 activity, whereas GATA1 activity is not repressed by ER binding to antagonist such as tamoxifen $(41,42)$.

These observations support the notion that estrogenic compounds cause apoptosis and repression of GATA1 target gene expression due to interaction of GATA1 and ligand-bound ER. If such interactions between GATA1 and ER occur in the GATA1 binding sites present in the promoter of Prx 5 gene, one of GATA1 target genes as demonstrated in our present study, regulation of Prx 5 may depend upon the ER ligand. For example, exposure to $\mathrm{E}_{2}$ may eliminate the inhibitory effects of GATA1 on Prx 5 gene expression; therefore, Prx 5 would be able to perform its role in protecting cells from oxidative stress and promoting cell survival in ER-positive breast cells. On the other hand, anti-estrogen agents may result in cell death or apoptosis in the same cells. Use of the anti-estrogen reagent tamoxifen, the drug of choice for treating ER-positive breast cancer, is seriously limited by the development of resistance $(4,48,49)$. The mechanisms of tamoxifen resistance are still poorly understood. We believe that our findings will help elucidate the molecular mechanism of tamoxifen resistance since the protective activity of Prx5 can be inhibited by tamoxifeninduced changes in binding of ER, GATA-ER interaction, and GATA1-mediated repression of Prx5 gene expression leading to survival from anti-estrogen challenge in ER-positive breast cancer cells.

In summary, the regulation of Prx5 via GATA1 repression and the protective function of Prx 5 in breast cancer cells have been demonstrated. Although the function of GATA1 has previ- ously been studied, mainly in erythropoiesis, the present data indicate that GATA1 may also decrease Prx5 gene expression in breast cancer cells. This novel regulation mechanism of gene expression may expand our understanding of Prx 5 functions in mammary carcinogenesis and anti-estrogen resistance.

\section{Acknowledgements}

This study was supported by the Basic Science Research Program through the National Research Foundation of Korea grants awarded to M. Chang (KRF-2010-0024998) and The National Research Foundation of Korea (NRF) grant funded by the Korean government (MEST) (No. 2011-0001382) to J.H. Park.

\section{References}

1. Beral V: Breast cancer and hormone-replacement therapy in the Million Women Study. Lancet 362: 419-427, 2003.

2. Seo MS, Kang SW, Kim K, Baines IC, Lee TH and Rhee SG: Identification of a new type of mammalian peroxiredoxin that forms an intramolecular disulfide as a reaction intermediate. $\mathrm{J}$ Biol Chem 275: 20346-20354, 2000.

3. Chang XZ, Li DQ, Hou YF, et al: Identification of the functional role of peroxiredoxin 6 in the progression of breast cancer. Breast Cancer Res Treat 9: R76, 2007.

4. Clarke R, Liu MC, Bouker KB, et al: Antiestrogen resistance in breast cancer and the role of estrogen receptor signaling. Oncogene 22: 7316-7339, 2003.

5. Egler RA, Fernandes E, Rothermund K, et al: Regulation of reactive oxygen species, DNA damage, and c-Myc function by peroxiredoxin 1. Oncogene 24: 8038-8050, 2005.

6. Neumann CA, Krause DS, Carman CV, et al: Essential role for the peroxiredoxin Prdx1 in erythrocyte antioxidant defence and tumour suppression. Nature 424: 561-565, 2003.

7. Radyuk SN, Michalak K, Klichko VI, Benes J and Orr WC: Peroxiredoxin 5 modulates immune response in Drosophila. Biochim Biophys Acta 1800: 1153-1163, 2010.

8. Lee TH, Kim SU, Yu SL, et al: Peroxiredoxin II is essential for sustaining life span of erythrocytes in mice. Blood 101: 5033-5038, 2003.

9. Yang CS, Lee DS, Song CH, et al: Roles of peroxiredoxin II in the regulation of proinflammatory responses to LPS and protection against endotoxin-induced lethal shock. J Exp Med 204: 583-594, 2007.

10. Kumin A,Huber C, Rulicke T, Wolf E and Werner S: Peroxiredoxin 6 is a potent cytoprotective enzyme in the epidermis. Am J Pathol 169: 1194-1205, 2006.

11. Kropotov A, Sedova V, Ivanov V, et al: A novel human DNAbinding protein with sequence similarity to a subfamily of redox proteins which is able to repress RNA-polymerase-III-driven transcription of the Alu-family retroposons in vitro. Eur J Biochem 260: 336-346, 1999.

12. Knoops B, Clippe A, Bogard C, et al: Cloning and characterization of AOEB166, a novel mammalian antioxidant enzyme of the peroxiredoxin family. J Biol Chem 274: 30451-30458, 1999.

13. Declercq JP and Evrard C: A twinned monoclinic crystal form of human peroxiredoxin 5 with eight molecules in the asymmetric unit. Acta Crystallogr D Biol Crystallogr 57: 1829-1835, 2001.

14. Nguyen-Nhu NT, Berck J, Clippe A, et al: Human peroxiredoxin 5 gene organization, initial characterization of its promoter and identification of alternative forms of mRNA. Biochim Biophys Acta 1769: 472-483, 2007.

15. Shiota M, Izumi H, Miyamoto N, et al: Ets regulates peroxiredoxin1 and 5 expressions through their interaction with the high-mobility group protein B1. Cancer Sci 99: 1950-1959, 2008.

16. Kropotov AV, Grudinkin PS, Pleskach NM, Gavrilov BA, Tomilin NV and Zhivotovsky B: Downregulation of peroxiredoxin $\mathrm{V}$ stimulates formation of etoposide-induced double-strand DNA breaks. FEBS Lett 572: 75-79, 2004.

17. Banmeyer I, Marchand C, Clippe A and Knoops B: Human mitochondrial peroxiredoxin 5 protects from mitochondrial DNA damages induced by hydrogen peroxide. FEBS Lett 579: 2327-2333, 2005. 
18. Kropotov A, Gogvadze V, Shupliakov O, et al: Peroxiredoxin V is essential for protection against apoptosis in human lung carcinoma cells. Exp Cell Res 312: 2806-2815, 2006.

19. Radyuk SN, Michalak K, Klichko VI, et al: Peroxiredoxin 5 confers protection against oxidative stress and apoptosis and also promotes longevity in Drosophila. Biochem J 419: 437-445, 2009.

20. Karihtala P, Mantyniemi A, Kang SW, Kinnula VL and Soini Y: Peroxiredoxins in breast carcinoma. Clin Cancer Res 9: 3418-3424, 2003.

21. Takabatake D, Fujita T, Shien T, et al: Tumor inhibitory effect of gefitinib (ZD1839, Iressa) and taxane combination therapy in EGFR-overexpressing breast cancer cell lines (MCF7/ADR, MDA-MB-231). Int J Cancer 120: 181-188, 2007.

22. Brunner N, Frandsen TL, Holst-Hansen C, et al: MCF7/LCC2: a 4-hydroxytamoxifen resistant human breast cancer variant that retains sensitivity to the steroidal antiestrogen ICI 182,780. Cancer Res 53: 3229-3232, 1993.

23. Mehta K: High levels of transglutaminase expression in doxorubicin-resistant human breast carcinoma cells. Int J Cancer 58 400-406, 1994.

24. Murphy MP: How mitochondria produce reactive oxygen species. Biochem J 417: 1-13, 2009.

25. Fridovich I: Fundamental aspects of reactive oxygen species, or what's the matter with oxygen? Ann N Y Acad Sci 893: 13-18, 1999.

26. Kamata $\mathrm{H}$ and Hirata $\mathrm{H}$ : Redox regulation of cellular signalling. Cell Signal 11: 1-14, 1999.

27. Sauer H, Wartenberg M and Hescheler J: Reactive oxygen species as intracellular messengers during cell growth and differentiation. Cell Physiol Biochem 11: 173-186, 2001.

28. Circu ML and Aw TY: Reactive oxygen species, cellular redox systems, and apoptosis. Free Radic Biol Med 48: 749-762, 2010.

29. Morise Z, Ueda M, Kitajima M, Epstein CJ, Granger DN and Grisham MB: Reactive oxygen species and vascular cell adhesion molecule-1 in distant organ failure following bile duct obstruction in mice. Dig Dis Sci 47: 607-613, 2002.

30. Avery SV: Molecular targets of oxidative stress. Biochem J 434: 201-210, 2011

31. Bartosz G: Reactive oxygen species: destroyers or messengers? Biochem Pharmacol 77: 1303-1315, 2009.

32. Maccarrone $M$ and Ullrich V: Redox regulation in disease and ageing. Cell Death Differ 11: 949-951, 2004.

33. Clerkin JS, Naughton R, Quiney C and Cotter TG: Mechanisms of ROS modulated cell survival during carcinogenesis. Cancer Lett 266: 30-36, 2008.

34. Kim YJ, Ahn JY, Liang P, Ip C, Zhang Y and Park YM: Human Prx1 gene is a target of Nrf2 and is up-regulated by hypoxia/reoxygenation: implication to tumor biology. Cancer Res 67: 546-554, 2007.

35. Graves JA, Metukuri M, Scott D, Rothermund K and Prochownik EV: Regulation of reactive oxygen species homeostasis by peroxiredoxins and c-Myc. J Biol Chem 284: 6520-6529, 2009.
36. Mikhailov VM, Kropotov AV, Zelenin AV, et al: [The BCL-xL and ACR-1 genes promote differentiation and reduce apoptosis in muscle fibers of mdx mice]. Genetika 38: 1445-1450, 2002.

37. Yuan J, Murrell GA, Trickett A, Landtmeters M, Knoops B and Wang MX: Overexpression of antioxidant enzyme peroxiredoxin 5 protects human tendon cells against apoptosis and loss of cellular function during oxidative stress. Biochim Biophys Acta 1693: 37-45, 2004.

38. Banmeyer I, Marchand C, Verhaeghe C, Vucic B, Rees JF and Knoops B: Overexpression of human peroxiredoxin 5 in subcellular compartments of Chinese hamster ovary cells: effects on cytotoxicity and DNA damage caused by peroxides. Free Radic Biol Med 36: 65-77, 2004

39. Aprelikova O, Wood M, Tackett S, Chandramouli GV and Barrett JC: Role of ETS transcription factors in the hypoxiainducible factor-2 target gene selection. Cancer Res 66: 5641-5647, 2006.

40. Bocchinfuso WP and Korach KS: Mammary gland development and tumorigenesis in estrogen receptor knockout mice. J Mal Gland Biol Neoplasia 2: 323-334, 1997.

41. Blobel GA and Orkin SH: Estrogen-induced apoptosis by inhibition of the erythroid transcription factor GATA-1. Mol Cell Biol 16: $1687-1694,1996$.

42. Blobel GA, Sieff CA and Orkin SH: Ligand-dependent repression of the erythroid transcription factor GATA-1 by the estrogen receptor. Mol Cell Biol 15: 3147-3153, 1995.

43. Chang M, Peng KW, Kastrati I, et al: Activation of estrogen receptor-mediated gene transcription by the equine estrogen metabolite, 4-methoxyequilenin, in human breast cancer cells. Endocrinology 148: 4793-4802, 2007.

44. Tsai SF, Martin DI, Zon LI, D'Andrea AD, Wong GG and Orkin SH: Cloning of cDNA for the major DNA-binding protein of the erythroid lineage through expression in mammalian cells. Nature 339: 446-451, 1989.

45. Evans T, Reitman M and Felsenfeld G: An erythrocyte-specific DNA-binding factor recognizes a regulatory sequence common to all chicken globin genes. Proc Natl Acad Sci USA 85: 5976-5980, 1988.

46. Newton A, Mackay J and Crossley M: The N-terminal zinc finger of the erythroid transcription factor GATA-1 binds GATC motifs in DNA. J Biol Chem 276: 35794-35801, 2001.

47. Tsang AP, Visvader JE, Turner CA, et al: FOG, a multitype zinc finger protein, acts as a cofactor for transcription factor GATA-1 in erythroid and megakaryocytic differentiation. Cell 90: 109-119, 1997.

48. Normanno N, Di Maio M, De Maio E, et al: Mechanisms of endocrine resistance and novel therapeutic strategies in breast cancer. Endocr Relat Cancer 12: 721-747, 2005.

49. Wang LH, Yang XY,Zhang X, et al: Disruption of estrogen receptor DNA-binding domain and related intramolecular communication restores tamoxifen sensitivity in resistant breast cancer. Cancer Cell 10: 487-499, 2006. 